\title{
Close-coupling approach to antiproton-impact breakup of molecular hydrogen
}

\author{
I. B. Abdurakhmanov, A. S. Kadyrov, D. V. Fursa, S. K. Avazbaev, and I. Bray \\ ARC Centre for Antimatter-Matter Studies, Curtin University, GPO Box U1987, Perth 6845, Australia
}

(Received 12 February 2014; published 28 April 2014)

\begin{abstract}
An $a b$ initio time-dependent convergent close-coupling approach to describing antiproton collisions with molecular hydrogen or the hydrogen molecular ion has been developed. The approach accounts for all possible orientations of the molecular target. Averaging over the molecular orientations is performed fully analytically. For the molecular hydrogen target calculated orientation-averaged total cross sections for single ionization and proton production are compared with several experiments over the energy range of 1-2000 keV. Results for single ionization are in good agreement with experiment, except for the region around the experimental maximum. For proton production reasonable agreement with experiment is observed above $40 \mathrm{keV}$.
\end{abstract}

DOI: 10.1103/PhysRevA.89.042706

PACS number(s): $34.10 .+\mathrm{x}, 34.50 . \mathrm{Gb}$

\section{INTRODUCTION}

Scattering of antiprotons from molecules is currently attracting great attention. One of the reasons is the relevance of the processes occurring during these collisions to radiotherapy and oncology (see, e.g., Refs. [1,2] and references therein). In addition, the clear understanding of antiproton interactions with atoms and molecules is important to the ALPHA collaboration at CERN that attempts to test the CPT invariance by forming and trapping antihydrogen [3] and study the gravitational behavior of antimatter at rest [4-6]. Also, the upcoming Facility for Antiproton and Ion Research (FAIR) [7] at GSI requires the precise knowledge of the collision mechanism between antiproton and molecular hydrogen, since $\mathrm{H}_{2}$ is expected to be one of the dominant residual-gas molecules.

Another motivation to study antiproton-impact ionization of molecules is of fundamental interest. The structure of molecular targets makes the underlying processes occur through mechanisms not observed for atomic targets. This point is supported by the latest experiments revealing unexpected features in the cross section for single nondissociative ionization of molecular hydrogen by low-energy $(2.4-9.9 \mathrm{keV})$ antiproton impact [8]. The measured single ionization cross section was shown to fall fast with decreasing energy. This was unlike the observed behavior of corresponding atomic cross sections and also was in sharp contrast to the results of prior theoretical calculations.

Among all of the ion-molecule scattering systems the antiproton-molecular hydrogen collision system is the simplest example due to the relative simplicity of the target and the absence of the electron capture channel. It is also the most convenient system to investigate molecular effects such as the influence of the vibrations and rotations of the molecule on the reaction dynamics. For this reason and also because of natural abundance of the target the antiproton scattering on molecular hydrogen is the most studied system both experimentally and theoretically. The cross sections for single nondissociative ionization of molecular hydrogen have been measured on three occasions; presently the experimental data exist on a wide energy range from $2.4 \mathrm{keV}$ to $1.6 \mathrm{MeV}$ [8-10]. Available theoretical approaches to the problem [11-15] can be classified into two categories. First, there are studies that employ a one-electron approximation with the use of model spherical effective potentials for the description of the target [11,12,14]. These approaches reproduce a reasonably accurate binding energy by tuning model parameters. Not surprisingly, these approaches are not sensitive to different molecular orientations. In addition, as the projectile energy decreases, these methods yield cross sections that fall off slowly. This is contrary to the experimental observation. Another class of studies uses a more accurate description of $\mathrm{H}_{2}$ where the contributions of electrons and nuclei of the molecular target are taken into account using the Born-Oppenheimer approximation [13-15]. These approaches obtain the orientationally averaged cross sections by averaging over only three orthogonal orientations. Calculations of Lühr and Saenz [13] support the overall behavior of the ionization cross sections at low energies that was observed in the experiment, but, there still remains a significant discrepancy. The approach of Pindzola and coworkers $[14,15]$ yields results that agree with experiment at the two lowest calculated energies. However, at higher energies this approach produces cross sections that are too low.

Recently we have developed a semiclassical timedependent convergent close-coupling approach to the antiproton-impact ionization of $\mathrm{H}_{2}$, which accounts for all possible orientations of the molecular target [16]. The approach is valid at all energies, and significantly improves the agreement between theory and experiment, though some discrepancies remain. We have presented quantitative confirmation of the experimentally observed phenomenon of target structureinduced suppression of the ionization cross section for lowenergy antiproton-molecular hydrogen collisions [8]. Here we present the details of the method, and additionally apply it to study antiproton collisions with another homonuclear, diatomic molecular target, $\mathrm{H}_{2}^{+}$. We will also describe the technique that allows for calculating cross sections for proton production in antiproton- $\mathrm{H}_{2}$ collisions by combining the ionization cross section results for $\mathrm{H}_{2}$ and $\mathrm{H}_{2}^{+}$targets.

The plan of the paper is as follows. In Sec. II, we describe our formalism, which starts from the full five-body timedependent Schrödinger equation for the antiproton-hydrogen molecule system. Details of the calculations are given in Sec. III and our results are presented in Sec. IV. Finally, in Sec. V we highlight the main findings and draw conclusions from this work. We use atomic units throughout unless otherwise specified. 


\section{FORMALISM}

The treatment of antiproton collisions with molecular hydrogen or the hydrogen molecular ion requires solving a multicenter many-body Coulomb problem. Therefore, it is crucial to choose an efficient approach. In these molecular targets the electrons are exposed to the superposition of two potentials with centers at the locations of the target protons. This causes the electronic wave function to be symmetric around the molecular axis. Therefore, for the purpose of target structure calculations it is best to utilize the body frame coordinates with the origin between the two protons and the $z^{\prime}$ axis pointing in the molecular axis direction $\boldsymbol{d}$. The vector $\boldsymbol{d}$ can be chosen to be the position of any of the protons relative to the other one. At the same time the scattering equations have to be formulated in the laboratory frame with the $z$ axis along the antiproton incident direction, since they directly lead to the experimental observables. The time-dependent, nonrelativistic, Schrödinger equation of a many-body system consisting of the incident antiproton $\bar{p}$, two target protons, and one $\left(\mathrm{H}_{2}^{+}\right)$or two $\left(\mathrm{H}_{2}\right)$ target electrons is

$$
H \Psi=i \frac{\partial \Psi}{\partial t}
$$

where the total Hamiltonian $H=H_{\mathrm{T}}+V$ is the sum of the target Hamiltonian $H_{\mathrm{T}}$ and the Coulombic interaction between the projectile and the target $V$. The target Hamiltonian can be written as a sum of nuclear and electronic parts as

$$
H_{\mathrm{T}}=H_{\mathrm{nucl}}+H_{\mathrm{elec}} \text {. }
$$

Here the nuclear part is

$$
H_{\text {nucl }}=-\frac{1}{2 M} \nabla_{\boldsymbol{R}_{1}}^{2}-\frac{1}{2 M} \nabla_{\boldsymbol{R}_{2}}^{2}+\frac{1}{d}
$$

with $M$ being the mass of a proton. The electronic part is a function of position vectors of the nuclei $\left(\boldsymbol{R}_{\boldsymbol{n}}\right)$ and electrons $\left(\boldsymbol{r}_{i}\right)$

$H_{\mathrm{elec}}=-\frac{1}{2} \sum_{i=1}^{N_{\mathrm{e}}} \nabla_{\boldsymbol{r}_{i}}^{2}-\sum_{n=1}^{2} \sum_{i=1}^{N_{\mathrm{e}}} \frac{1}{\left|\boldsymbol{r}_{i}-\boldsymbol{R}_{n}\right|}+\sum_{i=1}^{N_{\mathrm{e}}} \sum_{j>i}^{N_{\mathrm{e}}} \frac{1}{\left|\boldsymbol{r}_{i}-\boldsymbol{r}_{j}\right|}$,

where $N_{\mathrm{e}}=2$ for molecular hydrogen $\mathrm{H}_{2}$ and 1 for the hydrogen molecular ion $\mathrm{H}_{2}^{+}$. Finally, the interaction potential between the antiproton and the molecular target is

$$
V=-\frac{1}{|\boldsymbol{R}-\boldsymbol{d} / 2|}-\frac{1}{|\boldsymbol{R}+\boldsymbol{d} / 2|}+\sum_{i=1}^{N_{\mathrm{e}}} \frac{1}{\left|\boldsymbol{R}-\boldsymbol{r}_{i}\right|},
$$

where $\boldsymbol{R}$ is the position vector of the antiproton in the laboratory frame (see Fig. 1).

Solving the Schrödinger Eq. (1) with many degrees of freedom associated with nuclear and electronic motion of the target directly is extremely difficult. However, because of their much larger mass, the nuclei in a molecule move much slower than the electrons. This implies that the electrons can almost immediately adjust their positions to a changed nuclear configuration. In addition, we assume that the rate of the molecular oscillations is much smaller than the speed of the projectile $v$. With these assumptions we can express the

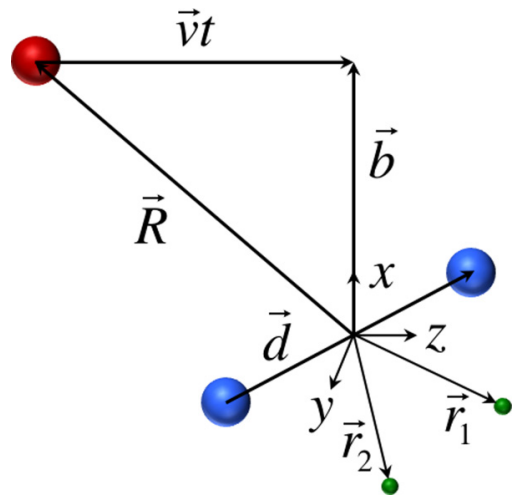

FIG. 1. (Color online) Sketch of the laboratory frame. The $z$ axis is parallel to the incident antiproton direction.

total wave function in a form where its nuclear and electronic variable dependent parts are separated, i.e.,

$$
\Psi(t, \boldsymbol{r}, \boldsymbol{R}, \boldsymbol{d})=\psi(t, \boldsymbol{r}, \boldsymbol{R}) \chi_{v j m}(\boldsymbol{d}),
$$

where $\chi_{v j m}(\boldsymbol{d})=\tilde{\chi}_{v j}(d) Y_{j m}\left(\theta_{d}, \phi_{d}\right)$ are the molecular wave functions representing the dependence on the internuclear distance within the target and $\psi$ is the electronic wave function. Here and elsewhere $\boldsymbol{r}$ collectively denotes the position vectors of all target electrons $\left(\boldsymbol{r}=\left\{\boldsymbol{r}_{\mathbf{1}}, \boldsymbol{r}_{\mathbf{2}}\right\}\right.$ for $\mathrm{H}_{2}$ and $\boldsymbol{r}=\left\{\boldsymbol{r}_{\mathbf{1}}\right\}$ for $\mathrm{H}_{2}^{+}$). Although Eq. (6) assumes the constant internuclear distance within the target throughout the collision, we will later investigate the effect of molecular oscillations using the Franck-Condon principle [17]. With the total wave function defined this way we can first solve the electronic problem with fixed nuclei:

$$
\left(H_{\mathrm{elec}}+V\right) \psi(t, \boldsymbol{r}, \boldsymbol{R})=i \frac{\partial \psi(t, \boldsymbol{r}, \boldsymbol{R})}{\partial t},
$$

and then use the solution that depends parametrically on the nuclear coordinates to restore the total wave function $\Psi$. The wave functions and corresponding energies for the rovibrational motion of the molecular target, $\chi_{v j m}(\boldsymbol{d})$ and $E_{v j}^{\text {nucl }}$, can be found from the following equation that also stems from Eq. (1):

$$
\left(H_{\text {nucl }}+E_{f}^{\text {elec }}\right) \chi_{v j m}(\boldsymbol{d})=E_{v j}^{\text {nucl }} \chi_{\nu j m}(\boldsymbol{d}),
$$

where $E_{f}^{\mathrm{elec}}$ is the total electronic energy of the final target state.

In this work we neglect the rotational degrees of freedom of the molecular target and consider the total wave function as

$$
\Psi(t, \boldsymbol{r}, \boldsymbol{R}, \boldsymbol{d})=\frac{1}{\sqrt{4 \pi}} \psi(t, \boldsymbol{r}, \boldsymbol{R}) \tilde{\chi}_{\nu 00}(d) \equiv \psi(t, \boldsymbol{r}, \boldsymbol{R}) \tilde{\chi}_{v}(d),
$$

where $v$ is the vibrational quantum number.

\section{A. Scattering equations}

In this section we will describe the method of solving the electronic part of the problem with fixed nuclei [Eq. (7)]. We use the semiclassical impact-parameter approach. However, the molecular target electron(s) is(are) treated fully quantum mechanically. The incident antiproton is assumed to be moving along a straight-line trajectory $\boldsymbol{R}(t)=\boldsymbol{b}+\boldsymbol{v} t$, where the 
impact parameter $\boldsymbol{b}$ points along the $x$ axis in the laboratory frame (see Fig. 1).

Following the ideas of the convergent close-coupling method we expand the electronic scattering wave function in terms of a certain set of target pseudostates $\Phi_{f}$ according to

$$
\psi(t, \boldsymbol{r}, \boldsymbol{R}=\boldsymbol{b}+\boldsymbol{v} t, \boldsymbol{d})=\sum_{f} A_{f}(t, \boldsymbol{b}, \boldsymbol{d}) \exp \left(-i \epsilon_{f} t\right) \Phi_{f}(\boldsymbol{r}, \boldsymbol{d}),
$$

where $\epsilon_{f}$ is the energy of the target electronic state $f$. The expansion coefficients $A_{f}(t, \boldsymbol{b}, \boldsymbol{d})$ define the probability for transitions into electronic bound and continuum states.

With this representation of the total scattering wave function the semiclassical Schrödinger equation can be transformed into a set of coupled-channel differential equations for the time-dependent coefficients $A_{f}(t, \boldsymbol{b}, \boldsymbol{d})$,

$$
\begin{aligned}
i \frac{d A_{f}(t, \boldsymbol{b}, \boldsymbol{d})}{d t}= & \sum_{i} A_{i}(t, \boldsymbol{b}, \boldsymbol{d})\left\langle\Phi_{f}|V(t, \boldsymbol{r}, \boldsymbol{b}, \boldsymbol{d})| \Phi_{i}\right\rangle \\
& \times \exp \left[i\left(\epsilon_{f}-\epsilon_{i}\right) t\right] .
\end{aligned}
$$

Equation (11) is solved with the initial conditions $A_{f}\left(t_{0}=\right.$ $-\infty, \boldsymbol{b}, \boldsymbol{d})=\delta_{f 0}$, as the target is initially in the ground state $\Phi_{0}$.

In contrast to atomic targets, which are spherically symmetric, for collisions with molecular targets solving just one set of coupled equations (11) is not sufficient. Here calculations for all molecular orientations have to be carried out in order to find orientationally averaged transition probabilities. A straightforward approach to solving Eq. (11) for all molecular orientations is computationally expensive. In previous studies that accounted for the multicenter nature of $\mathrm{H}_{2}$, therefore, the calculations were limited to only three orthogonal molecular orientations [13-15]. As far as the $\bar{p}-\mathrm{H}_{2}^{+}$ system is concerned, Sakimoto [18] accounted for $11 \times 5$ orientations for $\left(\theta_{d}, \phi_{d}\right)$. Later, Lühr and Saenz [19] compared their results obtained using only three orthogonal orientations with the results of Sakimoto [18], and found good agreement. They concluded that averaging over the three orientations was adequate for $\bar{p}-\mathrm{H}_{2}^{+}$. Whether this is the case for $\bar{p}-\mathrm{H}_{2}$ collisions as well, remains to be seen. To this end we have developed an alternative approach. Before giving details of the approach we need to describe how the target structure is treated.

\section{B. Treatment of molecular hydrogen and the hydrogen molecular ion}

As indicated above the target structure calculations are performed in the body frame (BF) coordinates that are denoted with primed variables (Fig. 2). For the description of the $\mathrm{H}_{2}$ electronic functions a single-center configuration-interaction (CI) expansion around the midpoint of the internuclear axis is used:

$$
\Phi_{f}^{\mathrm{BF}}\left(\boldsymbol{r}_{1}^{\prime}, \boldsymbol{r}_{2}^{\prime}, d\right)=\sum_{\alpha \beta} B_{\alpha \beta}^{f}(d) \phi_{\alpha}\left(\boldsymbol{r}_{1}^{\prime}\right) \phi_{\beta}\left(\boldsymbol{r}_{2}^{\prime}\right) .
$$



FIG. 2. (Color online) Sketch of the body frame. The $z^{\prime}$ axis is parallel to the internuclear axis of the target.

One-electron orbitals $\phi_{\alpha}$ are defined as

$$
\phi_{\alpha}(\boldsymbol{r})=\frac{\xi_{k l_{\alpha}}^{\left(\lambda_{l_{\alpha}}\right)}(r)}{r} Y_{l_{\alpha} m_{\alpha}}(\theta, \phi)
$$

where $Y_{l_{\alpha} m_{\alpha}}(\theta, \phi)$ are the spherical harmonics. One-electron functions $\xi_{k l}(r)$ are constructed using Laguerre polynomials as

$$
\begin{aligned}
\xi_{k l}^{\left(\lambda_{l}\right)}(r)= & \sqrt{\frac{\lambda_{l} r(k-1) !}{2(k+l)(k+2 l) !}}\left(\lambda_{l} r\right)^{l+1} \\
& \times \exp \left(-\frac{\lambda_{l} r}{2}\right) L_{k-1}^{2 l+1}\left(\lambda_{l} r\right),
\end{aligned}
$$

where $k$ ranges from 1 to the basis size $N_{l}$. The choice of the exponential fall-off parameter $\lambda_{l}$ will be discussed later. The expansion coefficients $B_{\alpha \beta}^{f}$ and target energy levels $\epsilon_{f}$ are found by diagonalizing the target Hamiltonian $H_{T}$. Presently, for the purpose of describing single ionization of $\mathrm{H}_{2}$, we restrict the upper limit of one of the indices in Eq. (12) in order to prevent the inner electron from ejecting. Specifically, we include only one-electron $\{1 s, 2 s, 2 p, 3 s, 3 p, 3 d\}$ orbitals for the description of the inner electron excitations. The other index representing the one-electron states of the outer electron can be as large as required to ensure converged results.

For the one-electron target, $\mathrm{H}_{2}^{+}$, the electronic functions have a simpler form:

$$
\Phi_{f}^{\mathrm{BF}}\left(\boldsymbol{r}_{1}^{\prime}, d\right)=\sum_{\alpha} B_{\alpha}^{f} \phi_{\alpha}\left(\boldsymbol{r}_{1}^{\prime}\right)
$$

Laboratory-frame electronic pseudostates $\Phi_{f}(\boldsymbol{r}, \boldsymbol{d})$ can be generated from the body-frame pseudostates (12) and (15) by rotating the latter into laboratory frame $\Phi_{f}(\boldsymbol{r}, \boldsymbol{d})=$ $\hat{D} \Phi_{f}^{\mathrm{BF}}(\boldsymbol{r}, d)$, where $\hat{D}$ is the rotation operator. In the following section we derive the matrix elements $\left\langle\Phi_{f}|V(t, \boldsymbol{r}, \boldsymbol{b}, \boldsymbol{d})| \Phi_{i}\right\rangle$ for both $\bar{p}-\mathrm{H}_{2}$ and $\bar{p}-\mathrm{H}_{2}^{+}$collision processes.

\section{Matrix elements}

Straightforward, laboratory-frame calculations of $\left\langle\Phi_{f}|V(t, \boldsymbol{r}, \boldsymbol{b}, \boldsymbol{d})| \Phi_{i}\right\rangle$ are computationally expensive, since the available body-frame target pseudostates first need to be converted into the laboratory frame before taking integrals. However, if we factor out the molecular-orientation-dependent part from the interaction potential (5), it will become irrelevant to what coordinate frame is used for taking the integrals over 
the configuration space of the electrons. The validity of the technique has been confirmed by obtaining exactly the same results as in the laboratory-frame calculations. The technique allows us to use the pseudostates defined in the body frame.

For $\bar{p}-\mathrm{H}_{2}$ collisions the matrix elements $\left\langle\Phi_{f}\left|V\left(t, \boldsymbol{r}_{1}, \boldsymbol{r}_{2}, \boldsymbol{b}, \boldsymbol{d}\right)\right| \Phi_{i}\right\rangle$ can be written as

$$
\begin{aligned}
& \left\langle\Phi_{f}\left|V\left(t, \boldsymbol{r}_{1}, \boldsymbol{r}_{2}, \boldsymbol{b}, \boldsymbol{d}\right)\right| \Phi_{i}\right\rangle \\
& =\int d \boldsymbol{r}^{\prime}{ }_{1} d \boldsymbol{r}_{2}^{\prime}{ }_{2} \Phi_{f}^{\mathrm{BF} *}\left(\boldsymbol{r}_{1}^{\prime}{ }_{1} \boldsymbol{r}_{2}^{\prime}, d\right) \\
& \quad \times\left(-\frac{1}{|\boldsymbol{R}-\boldsymbol{d} / 2|}-\frac{1}{|\boldsymbol{R}+\boldsymbol{d} / 2|}+\frac{1}{\left|\boldsymbol{R}-\boldsymbol{r}_{1}\right|}+\frac{1}{\left|\boldsymbol{R}-\boldsymbol{r}_{2}\right|}\right) \\
& \quad \times \Phi_{i}^{\mathrm{BF}}\left(\boldsymbol{r}_{1}^{\prime}, \boldsymbol{r}_{2}^{\prime}, d\right),
\end{aligned}
$$

where primed coordinates are relative to the body frame. Integration of the part corresponding to the interaction of the projectile with the target nuclei $I_{1}$ is straightforward due to the orthogonality of the target pseudostates and gives

$$
\begin{aligned}
I_{1}= & \int d \boldsymbol{r}_{1}^{\prime} d \boldsymbol{r}_{2}{ }_{2} \Phi_{f}^{\mathrm{BF} *}\left(\boldsymbol{r}_{1}^{\prime}, \boldsymbol{r}_{2}^{\prime}, d\right) \\
& \times\left(-\frac{1}{|\boldsymbol{R}-\boldsymbol{d} / 2|}-\frac{1}{|\boldsymbol{R}+\boldsymbol{d} / 2|}\right) \Phi_{i}^{\mathrm{BF}}\left(\boldsymbol{r}_{1}^{\prime}, \boldsymbol{r}_{2}^{\prime}, d\right) \\
= & -\delta_{f i}\left(\frac{1}{|\boldsymbol{R}-\boldsymbol{d} / 2|}+\frac{1}{|\boldsymbol{R}+\boldsymbol{d} / 2|}\right) .
\end{aligned}
$$

However, the remaining part $I_{2}$ is more challenging and involves partial-wave decomposition. By considering the symmetry with respect to interchanging $r_{1}$ and $r_{2}$ we can rewrite it as

$$
\begin{aligned}
I_{2}= & 2 \int d \boldsymbol{r}_{1}^{\prime} d \boldsymbol{r}_{2}^{\prime} \Phi_{f}^{\mathrm{BF} *}\left(\boldsymbol{r}_{1}^{\prime}, \boldsymbol{r}_{2}^{\prime}, d\right) \frac{1}{\left|\boldsymbol{R}-\boldsymbol{r}_{1}\right|} \Phi_{i}^{\mathrm{BF}}\left(\boldsymbol{r}_{1}^{\prime}, \boldsymbol{r}_{2}^{\prime}, d\right) \\
= & 8 \pi \sum_{\lambda \mu} \frac{1}{2 \lambda+1} Y_{\lambda \mu}^{*}(\hat{R}) \int d \boldsymbol{r}_{1}^{\prime} d \boldsymbol{r}_{2}{ }_{2} \Phi_{f}^{\mathrm{BF} *}\left(\boldsymbol{r}_{1}^{\prime}, \boldsymbol{r}_{2}{ }_{2}, d\right) \\
& \times \Phi_{i}^{\mathrm{BF}}\left(\boldsymbol{r}^{\prime}{ }_{1}, \boldsymbol{r}_{2}^{\prime}, d\right) v_{\lambda}\left(R, r_{1}\right) Y_{\lambda \mu}\left(\hat{r}_{1}\right)
\end{aligned}
$$

where

$$
v_{\lambda}\left(R, r_{1}\right)= \begin{cases}\frac{R^{\lambda}}{r_{1}^{\lambda+1}} & \text { if } R<r_{1} \\ \frac{r_{1}^{\lambda}}{R^{\lambda+1}} & \text { otherwise }\end{cases}
$$

With the target pseudostates defined as in Eq. (12) this term becomes

$$
\begin{aligned}
I_{2}= & 8 \pi \sum_{\lambda \mu} \sum_{\alpha \beta \gamma \delta} \frac{1}{2 \lambda+1} B_{\alpha \beta}^{f} B_{\gamma \delta}^{i} Y_{\lambda \mu}^{*}(\hat{R})\left\langle\phi_{\beta} \mid \phi_{\delta}\right\rangle \\
& \times \int d r_{1} \xi_{n_{\alpha} l_{\alpha}}\left(r_{1}\right) \xi_{n_{\gamma} l_{\gamma}}\left(r_{1}\right) v_{\lambda}\left(R, r_{1}\right) \\
& \times \int d \hat{r}_{1}^{\prime} Y_{l_{\alpha} m_{\alpha}}^{*}\left(\hat{r}_{1}^{\prime}\right) Y_{l_{\gamma} m_{\gamma}}\left(\hat{r}_{1}^{\prime}\right) Y_{\lambda \mu}\left(\hat{r}_{1}\right) .
\end{aligned}
$$

In order to perform the angular integration in Eq. (20), the coordinate $r_{1}$ originating from the partial-wave decomposition of the potential must be transformed to the body frame. $Y_{\lambda \mu}\left(\hat{r}_{1}\right)$ in the body frame becomes

$$
Y_{\lambda \mu}\left(\hat{r}_{1}\right)=\sum_{m} Y_{\lambda m}\left(\hat{r}_{1}^{\prime}\right) D_{\mu m}^{\lambda *}\left(\phi_{d}, \theta_{d}, 0\right),
$$

where $D_{\mu m}^{\lambda *}\left(\phi_{d}, \theta_{d}, 0\right)$ is the usual Wigner rotation matrix with $\phi_{d}$ and $\theta_{d}$ being the azimuthal and polar angles of the internuclear axis $\boldsymbol{d}$. We finally obtain

$$
\begin{aligned}
I_{2}= & 4 \sqrt{\pi} \sum_{\lambda \mu} D_{\mu m_{\alpha}-m_{\gamma}}^{\lambda *}\left(\phi_{d}, \theta_{d}, 0\right) Y_{\lambda \mu}^{*}(\hat{R}) \\
& \times \sum_{\alpha \beta \gamma \delta} B_{\alpha \beta}^{f} B_{\gamma \delta}^{i} \sqrt{\frac{2 l_{\gamma}+1}{(2 \lambda+1)\left(2 l_{\alpha}+1\right)}} C_{l_{\gamma} 0 \lambda 0}^{l_{\alpha} 0} C_{l_{\gamma} m_{\gamma} \lambda m_{\alpha}-m_{\gamma}}^{l_{\alpha} m_{\alpha}} \\
& \times\left\langle\phi_{\beta} \mid \phi_{\delta}\right\rangle \int d r_{1} \xi_{n_{\alpha} l_{\alpha}}\left(r_{1}\right) \xi_{n_{\gamma} l_{\gamma}}\left(r_{1}\right) v_{\lambda}\left(R, r_{1}\right),
\end{aligned}
$$

with $C_{\lambda \mu s q}^{\mathrm{LM}}$ denoting standard Clebsch-Gordan coefficients [20].

In order to combine this term with the term corresponding to the interaction of the projectile with the target nuclei, we also expand Eq. (17) into partial waves in a similar way as

$$
\begin{aligned}
& \frac{1}{|\boldsymbol{R}-\boldsymbol{d} / 2|}+\frac{1}{|\boldsymbol{R}+\boldsymbol{d} / 2|} \\
& =8 \pi \sum_{\lambda \mu} \frac{\bmod (\lambda, 2)}{2 \lambda+1} v_{\lambda}(R, d / 2) Y_{\lambda \mu}^{*}(\hat{R}) Y_{\lambda \mu}(\hat{d}) \\
& =4 \sqrt{\pi} \sum_{\lambda \mu} D_{\mu 0}^{\lambda *}\left(\phi_{d}, \theta_{d}, 0\right) Y_{\lambda \mu}^{*}(\hat{R}) \frac{\bmod (\lambda, 2)}{\sqrt{2 \lambda+1}} v_{\lambda}(R, d / 2),
\end{aligned}
$$

where $v_{\lambda}(R, d / 2)$ is defined by Eq. (19). Finally, using Eqs. (16), (22), and (23) we can write the matrix elements in the following form:

$$
\left\langle\Phi_{f}\left|V\left(t, \boldsymbol{r}_{1}, \boldsymbol{r}_{2}, \boldsymbol{b}, \boldsymbol{d}\right)\right| \Phi_{i}\right\rangle=\sum_{\lambda \mu} \mathcal{V}_{\lambda \mu}^{f i}(t, b, d) D_{\mu, m_{f}-m_{i}}^{\lambda *}\left(\phi_{d}, \theta_{d}, 0\right),
$$

where the molecular orientation-independent parts $\mathcal{V}_{\lambda \mu}^{f i}(t, b, d)$ are defined as

$$
\begin{aligned}
\mathcal{V}_{\lambda \mu}^{f i}(t, b, d)= & \frac{4 \sqrt{\pi} Y_{\lambda \mu}^{*}(\hat{R})}{\sqrt{2 \lambda+1}}\left(-\delta_{f i} \bmod (\lambda, 2) v_{\lambda}(R, d / 2)\right. \\
& +\sum_{\alpha \beta \gamma \delta} B_{\alpha \beta}^{f} B_{\gamma \delta}^{i} \sqrt{\frac{2 l_{\gamma}+1}{2 l_{\alpha}+1}} C_{l_{\gamma} 0 \lambda 0}^{l_{\alpha} 0} C_{l_{\gamma} m_{\gamma} \lambda m}^{l_{\alpha} m_{\alpha}}\left\langle\phi_{\beta} \mid \phi_{\delta}\right\rangle \\
& \left.\times \int d r_{1} \xi_{n_{\alpha} l_{\alpha}}\left(r_{1}\right) \xi_{n_{\gamma} l_{\gamma}}\left(r_{1}\right) v_{\lambda}\left(R, r_{1}\right)\right) .
\end{aligned}
$$

Expressing the interaction matrix elements in this form is important and will further help to eliminate the molecular orientation dependence from the scattering equations.

Antiproton collisions with the hydrogen molecular ion $\mathrm{H}_{2}^{+}$ are also modeled by the same equation (11). However, in this case the matrix elements should be calculated using the $\mathrm{H}_{2}^{+}$pseudostates and the appropriate interaction potential. 
Following similar steps the matrix elements for the $\bar{p}-\mathrm{H}_{2}^{+}$ collision can be shown to have the same form as Eq. (24). For this process the reduced matrix elements are independent of the molecular orientation and can be written as

$$
\begin{aligned}
\mathcal{V}_{\lambda \mu}^{f i}(t, b, d)= & \frac{4 \sqrt{\pi} Y_{\lambda \mu}^{*}(\hat{R})}{\sqrt{2 \lambda+1}}\left(-\delta_{f i} \bmod (\lambda, 2) v_{\lambda}(R, d / 2)\right. \\
& +\frac{1}{2} \sum_{\alpha \beta} B_{\alpha}^{f} B_{\beta}^{i} \sqrt{\frac{2 l_{\beta}+1}{2 l_{\alpha}+1}} C_{l_{\beta} 0 \lambda 0}^{l_{\alpha} 0} C_{l_{\beta} m_{\beta} \lambda m}^{l_{\alpha} m_{\alpha}} \\
& \left.\times \int d r \xi_{n_{\alpha} l_{\alpha}}\left(r_{1}\right) \xi_{n_{\beta} l_{\beta}}\left(r_{1}\right) v_{\lambda}\left(R, r_{1}\right)\right) .
\end{aligned}
$$

\section{Equation for the molecular orientation-independent part of the scattering amplitude}

We express the time-dependent coefficients in Eq. (11) in a form similar to (24) according to

$$
A_{f}(t, \boldsymbol{b}, \boldsymbol{d})=\sum_{\lambda \mu} \mathcal{A}_{\lambda \mu}^{f}(t, b, d) D_{\mu, m_{f}}^{\lambda *}\left(\phi_{d}, \theta_{d}, 0\right),
$$

where $\mathcal{A}_{\lambda \mu}^{f}(t, b, d)$ are the probability amplitudes independent of the molecular orientation. The expansion indices are limited by the maximum allowed total orbital angular momentum.

We substitute expansions (24) and (27) into Eq. (11) and, using the following identities for the Wigner functions,

$$
\begin{aligned}
& \int_{0}^{2 \pi} d \phi \int_{0}^{\pi} d \theta \sin \theta D_{M_{2} M_{2}^{\prime}}^{J_{2} *}\left(\phi_{d}, \theta_{d}, 0\right) D_{M_{1} M_{1}^{\prime}}^{J_{1} *}\left(\phi_{d}, \theta_{d}, 0\right) \\
& =\frac{4 \pi}{2 J_{2}+1} \delta_{J_{1} J_{2}} \delta_{M_{1} M_{2}} \delta_{M_{1}^{\prime} M_{2}^{\prime}} \\
& \int_{0}^{2 \pi} d \phi \int_{0}^{\pi} d \theta \sin \theta D_{M_{3} M_{3}^{\prime}}^{J_{3} *}\left(\phi_{d}, \theta_{d}, 0\right) \\
& \quad \times D_{M_{2} M_{2}^{\prime}}^{J_{2}}\left(\phi_{d}, \theta_{d}, 0\right) D_{M_{1} M_{1}^{\prime}}^{J_{1} *}\left(\phi_{d}, \theta_{d}, 0\right) \\
& =\frac{4 \pi}{2 J_{3}+1} C_{J_{1} M_{1} J_{2} M_{2}}^{J_{3} M_{3}} C_{J_{1} M_{1}^{\prime} J_{2} M_{2}^{\prime}}^{J_{3}},
\end{aligned}
$$

derive coupled differential equations for the molecularorientation-independent parts of the scattering amplitudes $\mathcal{A}_{\lambda \mu}^{f}(t, b, d)$ :

$$
\begin{aligned}
i \frac{d \mathcal{A}_{\lambda \mu}^{f}(t, b, d)}{d t}= & \sum_{i} \exp \left[i\left(\epsilon_{f}-\epsilon_{i}\right) t\right] \sum_{L M} \mathcal{A}_{L M}^{i}(t, b, d) \\
& \times \sum_{s q} \frac{2 \lambda+1}{2 L+1} C_{\lambda \mu s q}^{L M} C_{\lambda m_{f} s m_{i}-m_{f}}^{L m_{i}} \mathcal{V}_{s q}^{f i}(t, b, d) .
\end{aligned}
$$

This set of equations is solved subject to the initial conditions $\mathcal{A}_{\lambda \mu}^{f}\left(t_{0}=-\infty, b, d\right)=\delta_{f 0} \delta_{\lambda 0} \delta_{\mu 0}$. This boundary condition also implies that at infinite distance the antiproton does not feel the anisotropic nature of the molecular target.

\section{E. Calculation of cross sections}

Let us first consider antiproton collisions with molecular hydrogen. Cross sections for excitation and ionization are written in terms of the probabilities for the corresponding transitions between the initial and final states of $\mathrm{H}_{2}$. Those probabilities can be calculated via corresponding transition amplitudes, which are the overlap between the total wave function at $t=\infty$ and the final state of the target,

$$
\begin{aligned}
f_{f 0}(\boldsymbol{b})= & \frac{1}{\sqrt{4 \pi}} \int d \boldsymbol{d} d \boldsymbol{r}_{\mathbf{1}} d \boldsymbol{r}_{\mathbf{2}}\left[\psi\left(\boldsymbol{r}_{\mathbf{1}}, \boldsymbol{r}_{\mathbf{2}}, t=\infty, \boldsymbol{d}\right) \tilde{\chi}_{0}(d)\right]^{*} \\
& \times \Phi_{f}\left(\boldsymbol{r}_{\mathbf{1}}, \boldsymbol{r}_{\mathbf{2}}, \boldsymbol{d}\right) \tilde{\chi}_{v}^{r}(d) .
\end{aligned}
$$

Here, $\tilde{\chi}_{0}(d)$ describes the first vibrational level in the ground electronic state of the $\mathrm{H}_{2}$ molecule, whereas $\tilde{\chi}_{v}^{r}(d)$ describes the residual $\mathrm{H}_{2}^{+}$ion, in the vibrational state $v$ of the particular electronic state $f$. The factor $1 / \sqrt{4 \pi}$ is the normalization coefficient for molecular orientation averaging. The calculation of the integrals (30) is computationally expensive, since it involves generating a new set of pseudostates and solving the electronic problem for many internuclear distances $d$. For our collisions of interest, however, the dependence on $d$ is weak and almost linear as indicated in [18]. Therefore, it is possible to expand the electronic part of the integrand, $\left[\psi\left(\boldsymbol{r}_{\mathbf{1}}, \boldsymbol{r}_{\mathbf{2}}, t=\infty, \boldsymbol{d}\right)\right]^{*} \Phi_{f}\left(\boldsymbol{r}_{\mathbf{1}}, \boldsymbol{r}_{\mathbf{2}}, \boldsymbol{d}\right)$, into a Taylor series around the equilibrium distance $d_{0}$ and use only the first term $\left[\psi\left(\boldsymbol{r}_{\mathbf{1}}, \boldsymbol{r}_{\mathbf{2}}, t=\infty, \boldsymbol{d}_{\mathbf{0}}\right)\right]^{*} \Phi_{f}\left(\boldsymbol{r}_{\mathbf{1}}, \boldsymbol{r}_{\mathbf{2}}, \boldsymbol{d}_{\mathbf{0}}\right)$. With this we can factor out the integration over $d$ and write Eq. (30) in the following form:

$$
\begin{aligned}
f_{f 0}(\boldsymbol{b}) \approx & \frac{1}{\sqrt{4 \pi}} \int d^{2} d d \tilde{\chi}_{0}(d) \tilde{\chi}_{v}^{r}(d) \\
& \times \int d \varphi_{d} d \theta_{d} d \boldsymbol{r}_{1} d \boldsymbol{r}_{\mathbf{2}} \sin \theta_{d}\left[\psi\left(\boldsymbol{r}_{\mathbf{1}}, \boldsymbol{r}_{\mathbf{2}}, t=\infty, \boldsymbol{d}_{\mathbf{0}}\right)\right]^{*} \\
& \times \Phi_{f}\left(\boldsymbol{r}_{\mathbf{1}}, \boldsymbol{r}_{\mathbf{2}}, \boldsymbol{d}_{\mathbf{0}}\right)
\end{aligned}
$$

Given the orthogonality properties of the target electronic states the transition probabilities, which are the square of the absolute transition amplitudes, can be written as a product of two factors as

$$
\begin{aligned}
P_{f 0}(\boldsymbol{b})= & \frac{1}{4 \pi}\left|\int d^{2} d d \tilde{\chi}_{0}(d) \tilde{\chi}_{v}^{r}(d)\right|^{2} \\
& \times\left|\int d \varphi_{d} d \theta_{d} \sin \theta_{d} A_{f}\left(t=\infty, \boldsymbol{b}, \boldsymbol{d}_{\mathbf{0}}\right)\right|^{2} .
\end{aligned}
$$

The first factor $\left|\int d^{2} d d \tilde{\chi}_{0}(d) \tilde{\chi}_{v}^{r}(d)\right|^{2}$, also known as the Franck-Condon (FC) factor, carries information on probabilities for transitions between the vibrational levels in one electronic state and vibrational levels in any other electronic states of the molecule. Since we mainly concentrate on single ionization of $\mathrm{H}_{2}$ in the present work, we will need a list of FC factors for transitions between the first vibrational level in the ground state of the $\mathrm{H}_{2}$ molecule and all possible vibrational levels in the ground state of the residual ion $\mathrm{H}_{2}^{+}$. For this purpose we use the database of FC factors for molecule-ion reactions of $\mathrm{H}_{2}$ calculated by Wünderlich and Fantz [21] and Hunter et al. [22]. 
With this the total single ionization cross section independent of the molecular axis of the target is

$$
\begin{aligned}
\sigma_{\text {ion }}^{\nu}= & \sum_{f \in\left[\epsilon_{f} \geqslant 0\right]} \int d \boldsymbol{b} P_{f 0}(\boldsymbol{b}) \\
= & \left|\int d^{2} \mathrm{~d} d \tilde{\chi}_{0}(d) \tilde{\chi}_{v}^{r}(d)\right|^{2} \sum_{f \in\left[\epsilon_{f} \geqslant 0\right]} \sum_{\lambda \mu} \frac{2 \pi}{2 \lambda+1} \\
& \times \int_{0}^{\infty}\left|\mathcal{A}_{\lambda \mu}^{f}\left(t=+\infty, b, d_{0}\right)\right|^{2} b d b .
\end{aligned}
$$

This cross section describes single ionization of $\mathrm{H}_{2}$ where the residual ion $\mathrm{H}_{2}^{+}$in the ground electronic state is in the $v$ th vibrational level. At this point, if we are not concerned about the state of the residual ion and concentrate purely on the event of single ionization of the molecular target, we can sum up the ionization cross sections for all vibrational levels corresponding to both bound and continuum vibrational states. Since $\sum_{v}\left|\int d^{2} d d \tilde{\chi}_{0}(d) \tilde{\chi}_{v}^{r}(d)\right|^{2}=1$, the single ionization cross section can simply be calculated as

$$
\sigma_{\text {ion }}=\sum_{f \in\left[\epsilon_{f} \geqslant 0\right]} \sum_{\lambda \mu} \frac{2 \pi}{2 \lambda+1} \int_{0}^{\infty}\left|\mathcal{A}_{\lambda \mu}^{f}\left(t=+\infty, b, d_{0}\right)\right|^{2} b d b .
$$

However, experimental data for single ionization of $\mathrm{H}_{2}$ by antiproton impact are available only for the process where the residual $\mathrm{H}_{2}^{+}$ion stays bound. In other words, the experiment does not account for processes where the residual ion breaks apart into two protons before reaching the detector. Therefore, to take into account the possible experimental uncertainties we can use the following formula:

$$
\sigma_{\text {ion }}^{\exp }=\sum_{\nu \in\left[\epsilon_{v}<0\right]}\left|\int d^{2} d d \tilde{\chi}_{0}(d) \tilde{\chi}_{\nu}^{r}(d)\right|^{2} \sigma_{\text {ion }},
$$

where the summation is done over the negative energy $\left(\epsilon_{v}<0\right)$ vibrational levels.

Similarly, not being concerned about the fate of the residual ion, we can write the single ionization cross section, which is differential in the angular coordinates of the molecular axis, as

$$
\sigma_{\text {ion }}\left(\theta_{d}, \phi_{d}\right)=\sum_{f \in\left[\epsilon_{f} \geqslant 0\right]} \int d \boldsymbol{b}\left|A_{f}\left(t=\infty, \boldsymbol{b}, \boldsymbol{d}_{\mathbf{0}}\right)\right|^{2} .
$$

The consistency of the results for orientation-dependent cross sections calculated from solving Eq. (29) with those obtained from the direct solution of Eq. (11) has been checked.

For antiproton collisions with $\mathrm{H}_{2}^{+}$orientationally averaged and orientation-dependent cross sections are calculated the same way as in Eqs. (34) and (36), but using the scattering amplitudes, $\mathcal{A}_{\lambda \mu}^{f}\left(t=+\infty, b, d_{0}\right)$ and $A_{f}\left(t=\infty, \boldsymbol{b}, \boldsymbol{d}_{\mathbf{0}}\right)$ calculated for the $\bar{p}-\mathrm{H}_{2}^{+}$collision system. For the $\mathrm{H}_{2}^{+}$target we do not consider the influence of molecular oscillations and calculate the cross sections only in a pure fixed-nuclear approximation.

\section{F. Proton production in antiproton collisions with $\mathbf{H}_{2}$}

Measurements of cross sections for proton production in antiproton collisions with $\mathrm{H}_{2}$ were performed almost two decades ago [10]. However, the only theoretical work available so far significantly underestimates the experimental data [19]. Let us consider mechanisms in $\bar{p}-\mathrm{H}_{2}$ collisions that may lead to the production of protons. First, the double-electron ionization of the target results in the production of two protons. Second, the incident antiproton can ionize the molecular target, leaving the residual ion $\mathrm{H}_{2}^{+}$in (electronic) excited states that may further dissociate into atomic hydrogen and a proton. In the present work we use the so-called independent-event model (IEV). In this model all of the residual ions dissociate, since $\mathrm{H}_{2}^{+}$with the internuclear distance of $\mathrm{H}_{2}(d=1.4$ a.u. $)$ does not support any bound state. Thus, summing cross sections for processes that produce protons we can write

$$
\sigma_{\mathrm{H}^{+}}=2 \sigma_{\mathrm{di}}+\sigma_{\mathrm{ie}}
$$

where $\sigma_{\mathrm{di}}$ and $\sigma_{\mathrm{ie}}$ are the cross sections for double ionization and ionization with excitation, respectively.

To calculate these processes we follow the idea suggested in [23] and implemented in [19]. We implement the IEV model within the current time-dependent CCC method. In the IEV model the double ionization is considered as a two-step process. In the first and second steps single ionization of $\mathrm{H}_{2}$ and $\mathrm{H}_{2}^{+}$occur, respectively. Hence the total double ionization probability should be equal to the product of the probabilities for these single ionization processes:

$$
\sigma_{\mathrm{di}}=2 \pi \int P_{\mathrm{ion}}^{\mathrm{H}_{2}}(b) P_{\mathrm{ion}}^{\mathrm{H}_{2}+}(b) b d b .
$$

Similarly, the cross section for ionization with excitation can be expressed as

$$
\sigma_{\mathrm{ie}}=2 \pi \int P_{\mathrm{ion}}^{\mathrm{H}_{2}}(b) P_{\mathrm{exc}}^{\mathrm{H}_{2}+}(b) b d b .
$$

It should be noted that in calculating probabilities for $\mathrm{H}_{2}^{+}$the internuclear distance of the target is taken the same as for $\mathrm{H}_{2}$, i.e., $d_{0}=1.4$ a.u, since the events are happening at the same time. Clearly, calculating cross sections this way neglects any interference between the two effects. However, this appears to be a good approximation for calculations of double ionization in $\bar{p}$-He collisions [23].

\section{DETAILS OF CALCULATIONS}

The calculations for the $\mathrm{H}_{2}$ target presented below have been performed with $Z \equiv v t$ from -100 to +100 a.u. at all energies. In constructing the target basis we have included all $\mathrm{H}_{2}$ target states with the maximum value of angular momentum projection $m_{\max }$ equal to $l_{\max }$. To improve the accuracy of the calculations the Laguerre $1 s$ orbital was replaced with the $\mathrm{H}_{2}^{+} 1 s \sigma_{g}$ orbital, which was obtained via diagonalization of the $\mathrm{H}_{2}^{+}$Hamiltonian in the same Laguerre basis. The full set of antisymmetric two-electron configurations comprises two separate sets. The frozen-core $(1 s, n l m)$ configurations, where one electron is limited to the $1 s$ orbital of the $\mathrm{H}_{2}^{+}$ion, while the other occupies any of the Laguerre orbitals $(\mathrm{nlm})$. The other set takes all possible $\left(n^{\prime} l^{\prime} m^{\prime}, n l m\right)$ configurations with principle quantum numbers of Laguerre orbitals $n^{\prime}$ and $n \leqslant 3$. The frozen-core configurations allow for a square-integrable representation of the target continuum and coupling to the ionization channels in the scattering calculations. The primary 
TABLE I. Convergence of the total single ionization cross section with increasing $l_{\max }$ when $n_{\max }=20$.

\begin{tabular}{lcllll}
\hline \hline Energy $(\mathrm{keV})$ & $l=0$ & $l=1$ & $l=2$ & $l=3$ & $l=4$ \\
\hline 10 & 0.7762 & 0.1274 & 1.177 & 1.174 & 1.176 \\
50 & 0.3924 & 1.559 & 1.653 & 1.653 & 1.653 \\
100 & 0.2313 & 1.293 & 1.437 & 1.488 & 1.489 \\
\hline \hline
\end{tabular}

reason for including the $\left(n^{\prime} l^{\prime} m^{\prime}, n l m\right)$ configurations is to increase the accuracy in accounting for electron-electron correlations in the ground and low-lying excited states.

The accuracy of the final results for the orientationally averaged ionization cross section has been checked by performing calculations with several structure models that differ in the value of maximum orbital angular momentum $l_{\max }$ and number of one-electron Laguerre functions $N_{l}=n_{\max }-l$. The convergence studies have been carried out in the entire energy region considered in this work. We give typical examples at the projectile energies 5, 50, and $100 \mathrm{keV}$, i.e., at the position of the maximum in the experimentally measured total single ionization cross section (see below) and at some distance from the maximum on both sides.

First, we fix the basis parameter $n_{\max }$ at some large value and systematically increase the parameter $l_{\max }$ starting from 0 . Table I illustrates the convergence pattern of the total single ionization cross section (TSICS) with increasing $l_{\max }$, while $n_{\max }=20$ for each symmetry. One can see that at all considered energies convergence to within $0.2 \%$ is observed at $l_{\max }=4$. Next, we check whether $n_{\max }=20$ is sufficiently large in terms of the convergence of the cross section as a function of the principal quantum number of included states.

In Table II, we examine the convergence of the TSICS with $n_{\max }$ by setting $l_{\max }=4$. The convergence in the cross section when $n_{\max }$ changes from 5 to 20 is within $5 \%$. A similar rate of convergence has been achieved across the entire energy range with the target model consisting of 674 states, where $l_{\max }=4, N_{l}=20-l$, and a Laguerre basis exponential fall-off parameter $\lambda_{l}=2$. The ground-state energy obtained with this basis is -1.16497 a.u., which compares well with the accurate value of -1.1745 [24]. We have also checked the consistency of the calculations obtained using the present code with the previous fully quantum-mechanical results for the helium target [25], by taking the internuclear separation to zero (i.e., considering $\mathrm{He}$ as the united atom limit of $\mathrm{H}_{2}$ ).

For the calculations of $\bar{p}-\mathrm{H}_{2}^{+}$collisions with the target internuclear distances $d_{0}=1.4$ and 2.0 a.u. we use the basis with the same parameters. Because of the relative simplicity of $\mathrm{H}_{2}^{+}$compared to $\mathrm{H}_{2}$, the number of molecular target states is reduced to 430 .

TABLE II. Convergence of the TSICS with increasing $n_{\max }$ when $l_{\max }=4$.

\begin{tabular}{llccc}
\hline \hline Energy $(\mathrm{keV})$ & $n=5$ & $n=10$ & $n=15$ & $n=20$ \\
\hline 10 & 0.6382 & 1.154 & 1.156 & 1.146 \\
50 & 1.281 & 1.793 & 1.664 & 1.662 \\
100 & 1.130 & 1.627 & 1.469 & 1.479 \\
\hline \hline
\end{tabular}

Due to the partial-wave decomposition the dimension of the set of differential equations for the molecular-orientationindependent coefficients (29) is equal to the number of target channels multiplied by the number of partial waves representing the molecular target state. Thus, with $l_{\max }=4$ these numbers for $\mathrm{H}_{2}$ and $\mathrm{H}_{2}^{+}$are 81554 and 52 030, respectively.

\section{RESULTS OF CALCULATIONS}

\section{A. $\bar{p}-\mathrm{H}_{2}$ collisions}

In our recent publication [16] we have presented the energy dependence of the total cross section for single nondissociative ionization in $\bar{p}-\mathrm{H}_{2}$ collisions. The $\mathrm{CCC}$ results obtained using analytical averaging over all molecular orientations produced excellent agreement with experiment across the whole range of energies except for a small region from 20 to $90 \mathrm{keV}$. In this work we study the quality of approximate averaging that is obtained from a limited number of molecular orientations. This knowledge could be useful in the treatment of molecular collisions where the analytical averaging is not possible. We consider three perpendicular directions, $\left(\theta_{d}, \phi_{d}\right)=(0,0)$, $(\pi / 2,0)$, and $(\pi / 2, \pi / 2)$, as used by Lühr and Saenz [13] and Lee et al. [15]. In addition, we consider two other intermediate orientations $\left(\theta_{\mathrm{d}}, \phi_{\mathrm{d}}\right)=(\pi / 4,0)$ and $(-\pi / 4,0)$. The energy dependence of the total ionization cross sections for these five orientations is presented in Fig. 3. One can see that the curves differ considerably for different orientations. As the projectile energy increases above $100 \mathrm{keV}$ the results for molecular orientations lying on the plane with $\phi_{\mathrm{d}}=0$ converge to the same value. At lower energies there is considerable variation in the energy dependence of the cross sections. Compared to the results for other displayed orientations, cross sections for the molecular orientation $(\pi / 2, \pi / 2)$, which is perpendicular to the $\phi_{\mathrm{d}}=0$ plane are significantly larger at lower calculated energies and significantly smaller above $20 \mathrm{keV}$. A similar situation is observed in the calculations of Lühr and Saenz [19] for antiproton-impact ionization of $\mathrm{H}_{2}^{+}$. The results for the $(\pi / 2, \pi / 2)$ orientation are significantly different from the results for the other two, $(0,0)$ and $(\pi / 2,0)$, orientations.

In Fig. 4 we present the energy dependence of our single ionization cross sections averaged using two techniques

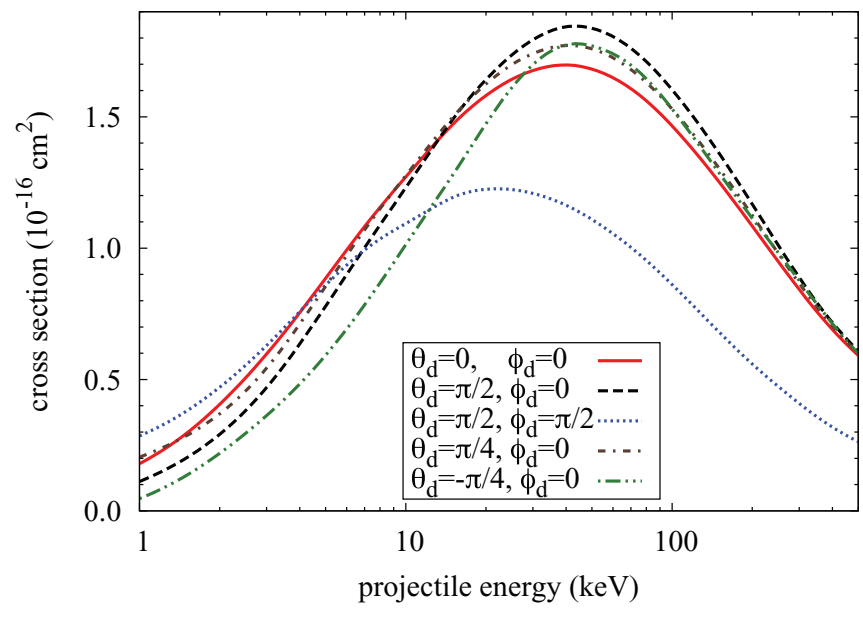

FIG. 3. (Color online) The total cross section for single ionization of $\mathrm{H}_{2}$ by antiprotons for different molecular orientations. 


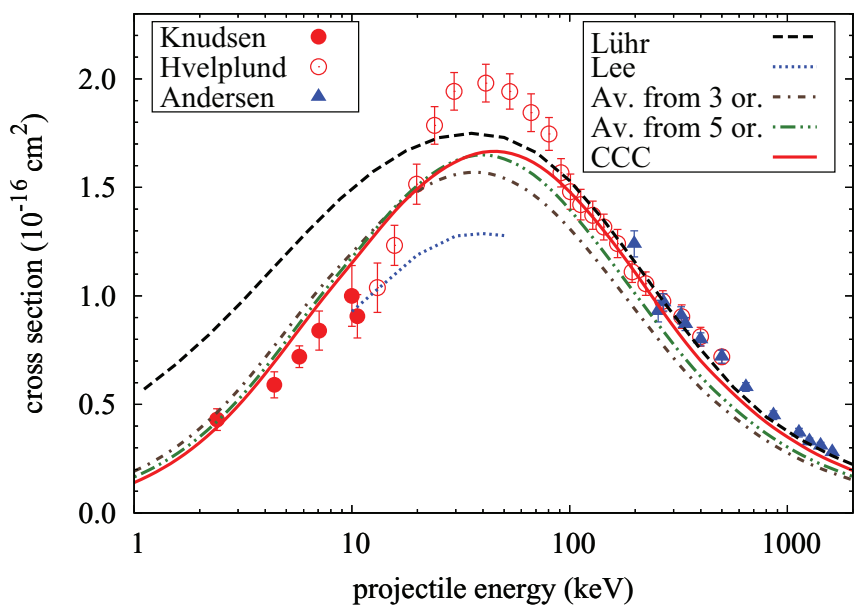

FIG. 4. (Color online) The total cross section for single ionization of $\mathrm{H}_{2}$ by antiprotons. Present $\mathrm{CCC}$ results obtained using various averaging techniques are compared with the experimental data of Andersen et al. [9], Hvelplund et al. [10], and Knudsen et al. [8], and two-electron calculations of Lee et al. [15] and Lühr and Saenz [13].

together with the experimental measurements $[8-10]$ and the two-electron calculations of Lühr and Saenz [13] and Lee et al. [15] obtained using three orthogonal molecular orientations. The results of Lühr and Saenz [13], being in excellent agreement with experiment at high energies clearly overestimates it at energies below $20 \mathrm{keV}$, whereas the results of Lee $e t$ al. [15] are generally lower and agree with experiment only at lower calculated energies. It should be noted that the calculations of Lee et al. [15] are for single ionization of the target with allowance for excited $\mathrm{H}_{2}^{+}$states. Their calculations without account of excited $\mathrm{H}_{2}^{+}$states (not shown) produced even smaller cross sections. Interestingly, our present results obtained from three orthogonal molecular orientations disagree with other calculations based on the three-orientation approximation. At the same time the present three-orientation results slightly overestimate our analytically averaged results at low energies, and significantly underestimate them at energies above $20 \mathrm{keV}$. In order to check whether the present results with approximate averaging come closer to the analytical results with increasing number of included molecular orientations we have considered two more intermediate orientations $\left[\left(\theta_{\mathrm{d}}, \phi_{\mathrm{d}}\right)=(\pi / 4,0)\right.$ and $\left.(-\pi / 4,0)\right]$. The averaged results over five orientations indeed are closer to the analytical results. As seen in Fig. 4 the difference between the analytical results and the results based on the three-orientation approximation can be as large as $20 \%$ at some energies. This suggests that the typical inclusion of only three orthogonal molecular orientations is not sufficient and an accurate method of orientation averaging is important. The present analytical averaging technique has certainly improved the agreement with experiment.

The discrepancy between theory and experiment in the energy region around $50 \mathrm{keV}$ still remains, and it is not likely to be related to the method of orientation averaging. As indicated in the previous section, after single nondissociative ionization, the residual ions of $\mathrm{H}_{2}^{+}$can dissociate before they reach the detector, provided they are in the vibrational continuum states.

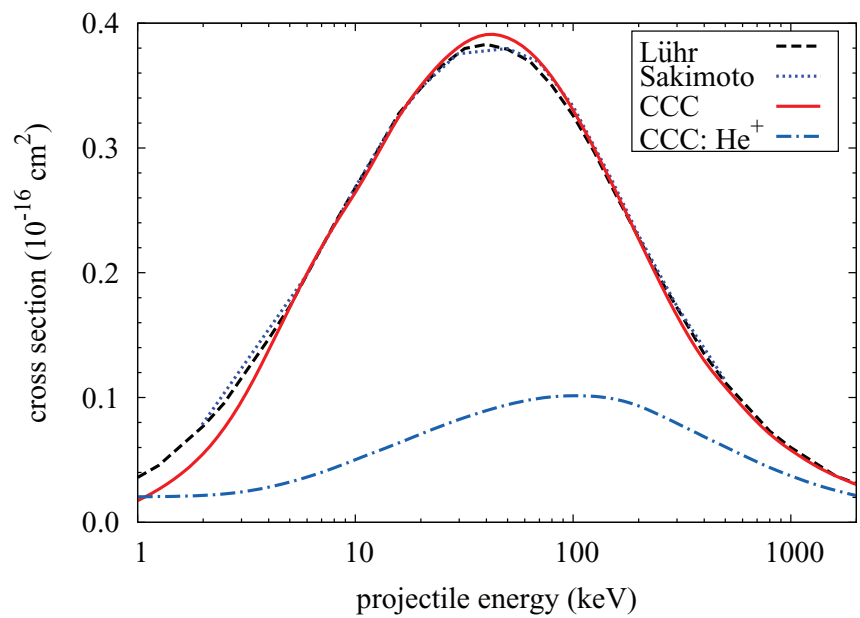

FIG. 5. (Color online) The total cross section for single ionization of $\mathrm{H}_{2}^{+}$by antiprotons. Present CCC results are compared with the two-center molecular-target calculations of Lühr and Saenz [19] and Sakimoto [18]. CCC results for the $\mathrm{He}^{+}$target are also presented.

Therefore, to evaluate the cross section that was measured in the experiment we have to use Eq. (35), which contains the Franck-Condon estimates for transitions from the ground vibrational level of $\mathrm{H}_{2}$ into all the negative energy vibrational levels of $\mathrm{H}_{2}^{+}$. The Franck-Condon factors were obtained from the database provided by Wünderlich and Fantz [21] and Hunter et al. [22]. The modified results are $1.7 \%$ lower than the results shown in Fig. 4. This way we find that at most 1.7\% of the residual $\mathrm{H}_{2}^{+}$ions may dissociate before reaching the detector. A similar estimate gives $0.5 \%$ for the $\mathrm{D}_{2}^{+}$ions used in the experiment. Thus, possible excitation of the vibrational continuum has little effect on our results.

\section{B. $\bar{p}-\mathrm{H}_{2}^{+}$collisions}

In Fig. 5 the present Franck-Condon results for total ionization obtained by analytical molecular-orientation averaging are compared to the corresponding results of Sakimoto [18] obtained from $11 \times 5$ orientations and those by Lühr and Saenz [19] obtained using only three orientations. While the previous two calculations $[18,19]$ are in good agreement with each other, the present results are slightly lower at low energies and slightly higher at the energies where the cross section has a maximum. Overall, the disagreement between the present results and the results of Lühr and Saenz [19] is surprisingly small in comparison with the disagreement observed for the molecular hydrogen target (Fig. 4). This observation is counterintuitive. The hydrogen molecular ion, $\mathrm{H}_{2}^{+}$, with the equilibrium internuclear distance $\left(d_{0}=2.0\right.$ a.u. $)$ larger than that of the neutral molecule, $\mathrm{H}_{2}\left(d_{0}=1.4\right.$ a.u. $)$, exhibits larger deviation from the spherical symmetry. Consequently, using only three molecular orientations for the purpose of calculating the orientationally averaged cross section for antiprotonimpact ionization of $\mathrm{H}_{2}^{+}$should have produced results that are in larger disagreement with the present analytically averaged results. This, however, is not the case.

In Fig. 5 we also present our results for antiproton-impact ionization of $\mathrm{He}^{+}$which is the united atom limit of $\mathrm{H}_{2}^{+}$. One can 
$\mathrm{Z}=-4$ a.u.
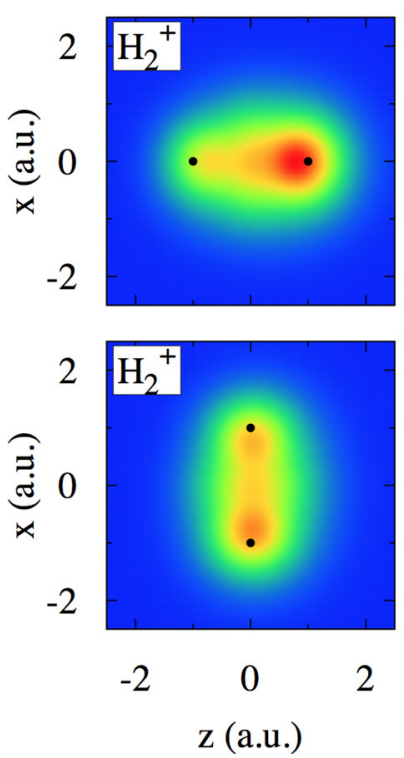

$\mathrm{Z}=-1$ a.u.
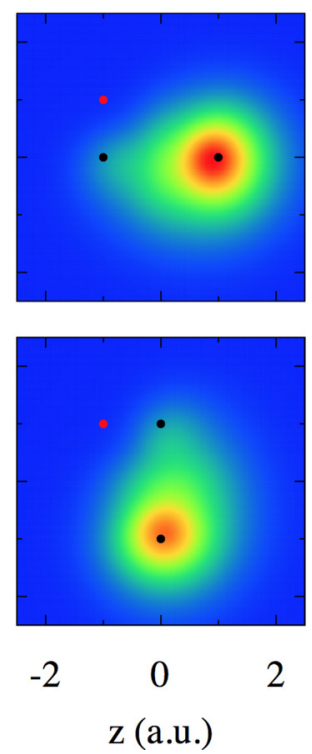

$\mathrm{Z}=0$ a.u.
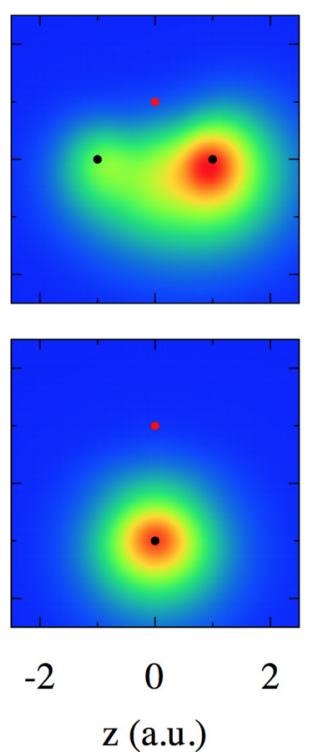

$\mathrm{Z}=1$ a.u.
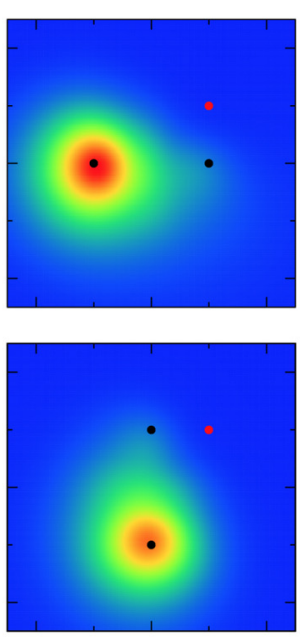

$-2$

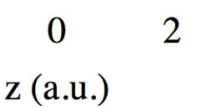

$\mathrm{Z}=4$ a.u.
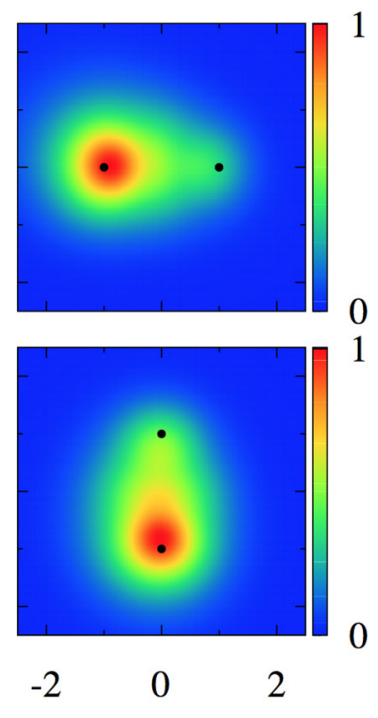

Z (a.u.)

FIG. 6. (Color online) The electron distribution dynamics in antiproton collisions with $\mathrm{H}_{2}^{+}$at $1 \mathrm{keV}$. The snapshots are taken at the impact parameter $b=1$ a.u. and several representative values of $Z=v t$. The corresponding projectile position is shown by red dots. The target nuclei are shown by black dots.

see that the ionization cross section for $\mathrm{He}^{+}$has a maximum near $100 \mathrm{keV}$ and slowly falls with decreasing impact energy, practically becoming flat at the lowest calculated energies. At all presented energies the cross section for ionization of $\mathrm{He}^{+}$ is significantly less than the cross section for ionization of $\mathrm{H}_{2}^{+}$ due to the large difference in the binding energies. One can see strong suppression of the $\mathrm{H}_{2}^{+}$cross section at low energies. The reason for this is the same as in the molecular hydrogen case. In order to see this point we have calculated the electron cloud distribution of the $\mathrm{H}_{2}^{+}$target during the collision similar to what we did for the $\bar{p}-\mathrm{H}_{2}$ process [16]. The snapshots shown in Fig. 6 reveal that the suppression mechanism is indeed the same, however, the electron movement from one target proton to another under the influence of the incoming antiproton is more pronounced than in the case of antiproton collisions with molecular hydrogen. This is related to the fact that the distance between the protons in the $\mathrm{H}_{2}^{+}$ion is larger than the corresponding distance within the $\mathrm{H}_{2}$ molecule. In other words, when the antiproton is close to one of the protons in $\mathrm{H}_{2}^{+}$, the electron has a chance to be bound to the other proton which is more isolated from Coulomb fields of the antiproton and the first proton.

\section{C. $\mathrm{H}^{+}$production in $\bar{p}-\mathrm{H}_{2}$ collisions}

In Fig. 7 the present CCC results for $\sigma_{\mathrm{di}}, \sigma_{\mathrm{ie}}$, and $\sigma_{\mathrm{H}^{+}}$are compared with the experimental data of Hvelplund et al. [10]. Also shown are the corresponding cross sections calculated by Lühr and Saenz [19]. In spite of the two-step approximation the present results agree reasonably well with experiment above $40 \mathrm{keV}$. Below $40 \mathrm{keV}$ our results are systematically higher than experiment, indicating that the IEV model becomes less reliable at low energies. In addition, it is worth mentioning that the mechanism of $\mathrm{H}^{+}$production due to ionization with excitation contributes more than twice compared to double ionization.

The results of Lühr and Saenz [19] are systematically lower than experiment at all available energies. The individual cross sections for double ionization and ionization with excitation of the target are also systematically lower than the corresponding present results. We emphasize that Lühr and Saenz [19] performed $\sigma_{\mathrm{H}^{+}}$calculations using an internuclear distance $d_{0}=2.0$ a.u. for $\mathrm{H}_{2}^{+}$and 1.4478 a.u. for $\mathrm{H}_{2}$, despite the fact that the two-step approximation requires using the same internuclear distance $d_{0}=1.4478$ a.u. for both targets. They

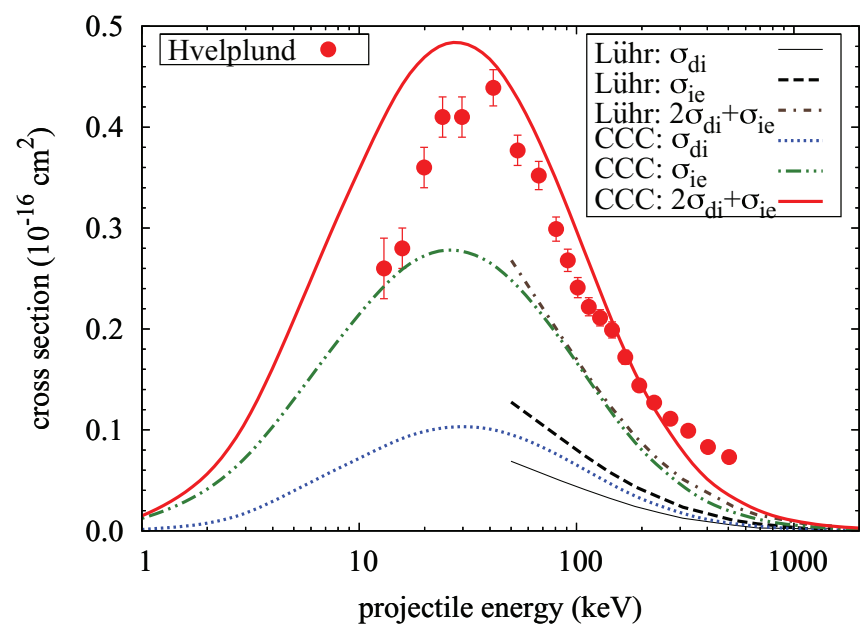

FIG. 7. (Color online) Cross sections leading for production of $\mathrm{H}^{+}$(see text) in antiproton collisions with $\mathrm{H}_{2}$. Present CCC results obtained within the two-step approximation (see text) are compared with the experimental data of Hvelplund et al. [10] and calculations of Lühr and Saenz [19]. 
indicated that similar calculations with $d_{0}=1.4478$ a.u. for $\mathrm{H}_{2}^{+}$ produced even lower cross sections.

\section{CONCLUSIONS}

Antiproton collisions with $\mathrm{H}_{2}$ have been studied using a time-dependent convergent close-coupling approach. While still taking advantage of the commonly used fixed-nuclei approximation, the present approach accounts for all possible orientations of the molecular target by analytically averaging over the molecular orientations. A set of scattering equations that are independent of the molecular orientation has been derived. A similar technique can be implemented in studies of projectile collisions with other molecular targets. The presented approach is valid at all energies, and significantly improves the agreement between theory and experiment for $\bar{p}-\mathrm{H}_{2}$ single ionization cross sections. The strong suppression of the single ionization cross section at low energies has been shown to be attributed solely to the structure of the molecular target. Studies of the time evolution of the electron cloud distribution during the antiproton collisions with atomic and molecular hydrogen showed that the electron, which would have gone into the continuum in the atomic hydrogen case, gets caught by one of the target protons in the molecular hydrogen case. A small region near the experimental maximum, where a discrepancy is observed needs further attention from both theorists and experimentalists.
In addition, to complete the picture of the full target breakup, the cross sections for ionization with excitation, double ionization, and proton production in antiproton-molecular hydrogen collisions have been calculated using a two-step approximation. Good agreement with experiment for proton production was found above $40 \mathrm{keV}$. Apparently, the two-step approximation employed in the calculations is problematic at lower energies. A more accurate treatment of the two-electron processes is yet to be developed.

The approach has been extended to study antiprotonimpact ionization of another homonuclear diatomic molecule, $\mathrm{H}_{2}^{+}$. Except for minor discrepancies the present results with analytical orientational averaging are in good agreement with previous studies that used only three orthogonal orientations of the $\mathrm{H}_{2}^{+}$target.

As a future direction, the study of proton collisions with molecular hydrogen is highly interesting. The mechanism of ionization, which is governed by the interplay of attractive Coulomb fields of three protons and also competes with the electron capture channel, certainly deserves attention.

\section{ACKNOWLEDGMENTS}

We thank Klaus Bartschat for careful reading of our manuscript and his helpful comments and suggestions. The work was supported by the Australian Research Council. We are grateful for access to the Australian National Computing Infrastructure Facility and its Western Australian node iVEC.
[1] N. Bassler, J. Alsner, G. Beyer, J. J. DeMarco, M. Doser, D. Hajdukovic, O. Hartley, K. S. Iwamoto, O. Jkel, H. V. Knudsen, S. Kovacevic, S. P. Mller, J. Overgaard, J. B. Petersen, T. D. Solberg, B. S. Mller, S. Vranjes, B. G. Wouters, and M. H. Holzscheiter, Radiother. Oncol. 86, 14 (2008).

[2] T. Kirchner and H. Knudsen, J. Phys. B 44, 122001 (2011).

[3] The ALPHA Collaboration, Nature (London) 483, 439 (2012).

[4] The ALPHA Collaboration and A. E. Charman, Nat. Commun. 4, 1785 (2013).

[5] The AEGIS Collaboration, Nucl. Instrum. Methods B 266, 351 (2008).

[6] P. Debu for the GBAR Collaboration, Hyperfine Interact. 212, 51 (2012).

[7] Facility for Antiproton and Ion Research [www.fair-center.org].

[8] H. Knudsen, H. A. Torii, M. Charlton, Y. Enomoto, I. Georgescu, C. A. Hunniford, C. H. Kim, Y. Kanai, H.-P. E. Kristiansen, N. Kuroda, M. D. Lund, R. W. McCullough, K. Tökesi, U. I. Uggerhøj, and Y. Yamazaki, Phys. Rev. Lett. 105, 213201 (2010).

[9] L. H. Andersen, P. Hvelplund, H. Knudsen, S. P. Moller, J. O. P. Pedersen, S. Tang-Petersen, E. Uggerhoj, K. Elsener, and E. Morenzoni, J. Phys. B 23, L395 (1990).

[10] P. Hvelplund, H. Knudsen, U. Mikkelsen, E. Morenzoni, S. P. Moller, E. Uggerhoj, and T. Worm, J. Phys. B 27, 925 (1994).

[11] A. Ermolaev, Hyperfine Interact. 76, 335 (1993).
[12] A. Lühr and A. Saenz, Phys. Rev. A 78, 032708 (2008).

[13] A. Lühr and A. Saenz, Phys. Rev. A 81, 010701 (2010).

[14] M. S. Pindzola, T.-G. Lee, and J. Colgan, J. Phys. B 43, 235201 (2010).

[15] T. G. Lee, M. S. Pindzola, and J. Colgan, J. Phys. B 45, 045203 (2012).

[16] I. B. Abdurakhmanov, A. S. Kadyrov, D. V. Fursa, and I. Bray, Phys. Rev. Lett. 111, 173201 (2013).

[17] W. Demtröder, Atoms, Molecules and Photons: An Introduction to Atomic-, Molecular- and Quantum Physics, Graduate Texts in Physics (Springer, New York, 2010).

[18] K. Sakimoto, Phys. Rev. A 71, 062704 (2005).

[19] A. Lühr and A. Saenz, Phys. Rev. A 80, 022705 (2009).

[20] D. A. Varshalovich, A. N. Moskalev, and V. K. Khersonskii, Quantum Theory of Angular Momentum, 1st ed. (World Scientific, Philadelphia, 1988).

[21] D. Wünderlich and U. Fantz, At. Data Nucl. Data Tables 97, 152 (2011).

[22] G. Hunter, A. W. Yau, and H. O. Pritchard, At. Data Nucl. Data Tables 14, 11 (1974).

[23] L. A. Wehrman, A. L. Ford, and J. F. Reading, J. Phys. B 29, 5831 (1996).

[24] T. E. Sharp, At. Data Nucl. Data Tables 2, 119 (1970).

[25] I. B. Abdurakhmanov, A. S. Kadyrov, D. V. Fursa, I. Bray, and A. T. Stelbovics, Phys. Rev. A 84, 062708 (2011). 